Ann. Génét. Sél. anim., I976, 8 (3), 331-342.

\title{
INTÉRET DE LA TRANSPLANTATION D'CEUFS POUR ACGROITRE L'EFFICACITÉ DE LA SÉLECTION DES BOVINS A VIANDE
}

\author{
J.-M. ELSEN et J.-C. MOCQUOT \\ Station de Génétique quantitative et appliquée, \\ Centre national de Recherches zootechniques, I. N.R. A., \\ 78350 Jouy en Josas
}

\section{RÉSUMÉ}

Nous avons étudié les possibilités offertes par les nouvelles techniques de superovulation et de transplantation des œufs, pour accroître le progrès génétique réalisable dans une souche de bovins de croisement terminal, quand les reproducteurs sont choisis sur leurs performances individuelles mesurées en station et que le nombre de places de contrôle est limité. Deux situations ont été comparées :

- une situation de référence, sans transplantation, où seuls les mâles sont contrôlés,

- une situation avec transplantation où une partie des places de station est réservée au contrôle des femelles.

Nous avons, pour des capacités de testage différentes, considéré des nombres väriables de mâles et de femelles contrôlés et sélectionnés comme reproducteurs et envisagé, pour la seconde situation, d'obtenir un nombre moyen de descendants par femelle donneuse plus ou moins important.

A capacité de testage fixée, les différentes situations ainsi simulées ont été comparées d'après le progrès génétique maximum qu'elles permettent d'espérer pour un accroissement donné du taux de consanguinité. Les résultats obtenus conduisent aux conclusions suivantes :

- L'intérêt de la transplantation est particulièrement notable pour des valeurs moyennes de l'accroissement du taux de consanguinité puisque entre 0,5 et I,5 p. roo par an le progrès génétique peut être doublé par rapport à la sitution de référence ; alors que la situation sans transplantation est préférable en dessous de $0,5 \mathrm{p}$. roo et qu'au-delà de $\mathrm{I}, 5 \mathrm{p}$. roo d'accroissement annuel du taux de consanguinité l'augmentation relative du progrès génétique n'est plus sensible.

- L'intérêt génétique de la transplantation n'est réellement sensible que pour des nombres limités d'œufs transplantés puisque, au-delà de 6 à 8 descendants par femelle donneuse, l'accroissement relatif du progrès génétique devient très faible.

- Enfin, la fraction optimale des places de statión à réserver au contrôle des mâles est toujours égale ou supérieure à $50 \mathrm{p}$. roo et augmente avec le nombre de taureaux sélectionnés. 


\section{INTRODUCTION}

Les techniques de superovulation et de transplantation d'œufs, dont on peut espérer qu'elles seront largement utilisables dans un avenir proche, présentent un intérêt certain pour l'amélioration génétique des bovins. Plusieurs auteurs ont déjà esquissé une réflexion à ce sujet (LAND et HrLL, I975 ; CunNINGHAM, r974 ; VISSAC, I974).

D'un point de vue général ces techniques peuvent permettre d'intervenir plus rapidement sur la création et la diffusion de matériel génétique, d'une part en réduisant l'intervalle de génération pour les voies mères-descendants (mères-fils et mèresfilles), d'autre part, en permettant un accroissement de l'intensité de sélection des femelles pour ces deux voies de transmission des gènes. L'exploitation du premier avantage pour l'utilisation rapide de schémas de croisement est évidente.

L'intérêt conjoint de ces deux avantages, pour la sélection, risque par contre de n'être appréciable que dans la mesure où les critères de sélection utilisés sont suffisamment précoces et précis. LAND et HrL,L (1975) ont étudié deux situations relatives, l'une au cas d'un critère maternel encore spéculatif (taux d'ovulation), l'autre au cas plus classique des caractères de croissance et de conformation pour la sélection des souches de croisement terminal. Ils ont analysé dans chacune de ces deux situations l'incidence de l'emploi des techniques de transplantation sur le progrès génétique et l'accroissement de consanguinité possibles dans une petite population conduite en monte naturelle et sélectionnée d'après la valeur individuelle des animaux mesurée en ferme.

La mise en œuvre de ces techniques nouvelles nous paraît plus particulièrement adaptée et utile dans le cas des schémas intégrés de sélection des souches de croisement terminal réalisés par les coopératives d'insémination du sud-ouest de la France, nous avons développé l'étude de L,AND et HILL en tenant compte des caractéristiques et contraintes propres à ces schémas. En particulier la nécessité croissante de sélectionner des caractères tels que l'indice de consommation implique un contrôle des animaux en station donc une limitation importante de la capacité de testage que l'on peut par ailleurs affecter dans des proportions variables aux mâles et aux femelles.

\section{I. - MATÉRIEL E'T MÉTHODES}

\section{I. - Schémas de sélection comparés et hypothèses}

Nous avons comparé deux schémas de sélection décrits sur la figure $\mathrm{r}$. Ces schémas sont basés sur un choix des reproducteurs (mâles dans un cas, mâles et femelles dans l'autre), d'après leurs performances mesurées en station à l'âge de 12 mois. Cette situation ne correspond pas à l'optimum qui est obtenu pour la sélection des souches de croisement terminal par application d'une deuxième étape de sélection sur la descendance croisée des taureaux (MocQuoT et FouLLEY, 1973). L'introduction d'un tel contrôle de descendance ne changerait pas les conclusions dans la mesure où l'efficacité de ce contrôle (coûts et recettes) serait comparable dans les deux cas. Nous n'avons pas non plus introduit de sélection des mâles sur l'aptitude à produire de la semence dans la mesure où nous ne sommes concernés que par le progrès génétique réalisé en race pure donc par le choix des pères à taureaux destinés à réaliser chacun un petit nombre d'inséminations. 
Il est bien évident que ce tri sur spermatogenèse s'impose pour l'utilisation en croisement des taureaux issus de station, et que dans ce cas une contrainte supplémentaire doit être introduite quant au nombre minimum de places à réserver aux mâles dans la station.
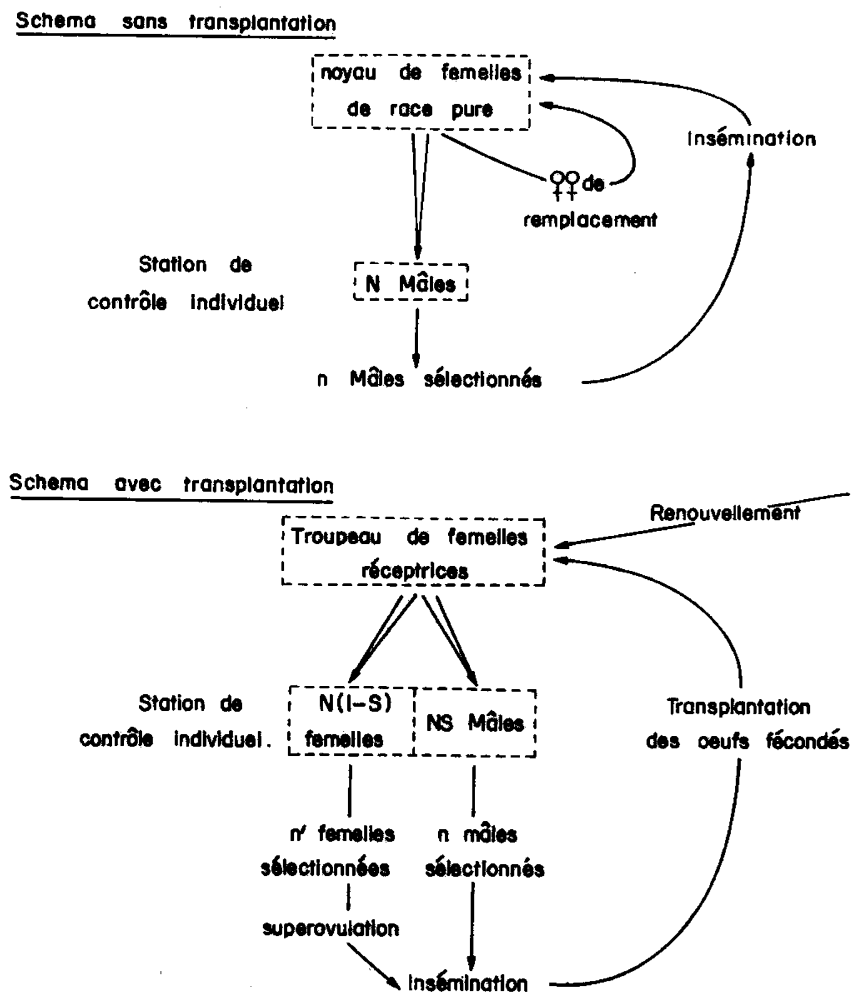

FIG. I. - Description des situations comparées

Description of the compared situations

Dans la situation de référence (sans transplantation) seuls les veaux mâles, produits par ur. noyau de femelles de race pure, entrent en station de contrôle individuel. A l'issue de ce contrôle ils sont sélectionnés sur leurs performances à 12 mois (croissance, conformation, indice de consommation). Les $n$ mâles retenus sont alors utilisés comme reproducteurs et dès l'âge de I5 mois pendant une année sur le noyau de femelles. Dans ce cas les femelles issues de ces taureaux ne sont pas contrôlées car presque toutes sont indispensables au renouvellement du noyau de race pure. Par ailleurs aucun choix des mères des veaux mâles entrés en station n'est possible dans la mesure où on souhaite, pour réduire les coûts au maximum, n'entretenir que le nombre minimum de femelles nécessaire à produire les mâles.

Dans la seconde situation envisagée l'utilisation des techniques de transplantation doit permettre d'accroître sensiblement les possibilités de sélection des femelles et donc d'envisager leur contrôle en station.

Nous leur avons réservé dans ce cas une fraction (I-S), variable, des $\mathrm{N}$ places disponibles en station et supposé que leur sélection se ferait sur le même critère, ayant la même héritabilité, que pour les mâles. Dès lors les $n^{\prime}$ femelles sélectionnées sont traitées pour la superovulation, inséminées entre 18 et 24 mois par les $n$ mâles retenus et les œufs récoltés sont transplantés dans des femelles réceptrices. On a supposé qu'au cours de ces 6 mois ces opérations sont réalisées deux fois pour chaque femelle, ce qui permet éventuellement de l'accoupler à 2 mâles différents. En l'absence de données plus précises nous n'avons retenu comme paramètre technique de transplantation que le nombre $(K)$ de descendants, nés vivants et susceptibles d'entrer en station, produits en moyenne par femelle donneuse. Ceci ne laisse rien présumer sur le nombre d'œufs 
récoltés par cycle, ni sur le nombre de femelles réceptrices nécessaires. Faute de ces informations on ne pourra pas pour comparer les deux situations faire intervenir les différences de conts de production des veaux obtenus.

Nous avons fait figurer sur le tableau I les valeurs des paramètres nécessaires à la réalisation des calculs. Les intervalles de génération considérés pour le premier schéma supposent un premier vêlage des femelles de race pure à 36 mois et un taux de renouvellement de 25 p. Ioo soit une durée moyenne de vie reproductive de quatre ans. Nous avons attribué plusieurs valeurs à un certain nombre de paramètres dont nous souhaitions étudier l'influence sur l'efficacité comparée des schémas de sélection envisagés. Il s'agit notamment de la capacité de testage (N) et de sa fraction (I - S) réservée aux femelles dans le second cas, du nombre de mâles retenus comme reproducteurs $(n)$ et du nombre de descendants $(K)$ par donneuse dans la mesure où il constitue le paramètre clé de cette étude et où sa valeur est encore très spéculative dans l'état actuel des techniques. Il faut cependant signaler que ce nombre est très lié au nombre de cycles de superovulation et transplantation envisagés pour chaque femelle donneuse et par conséquent à l'intervalle de génération pour les voies femelles-mâles et femelles-femelles.

\section{TABLEAU I}

Notation et valeurs admises pour les paramètres utilisés äans le modèle

Notation and values assumed for the parameters used in the model

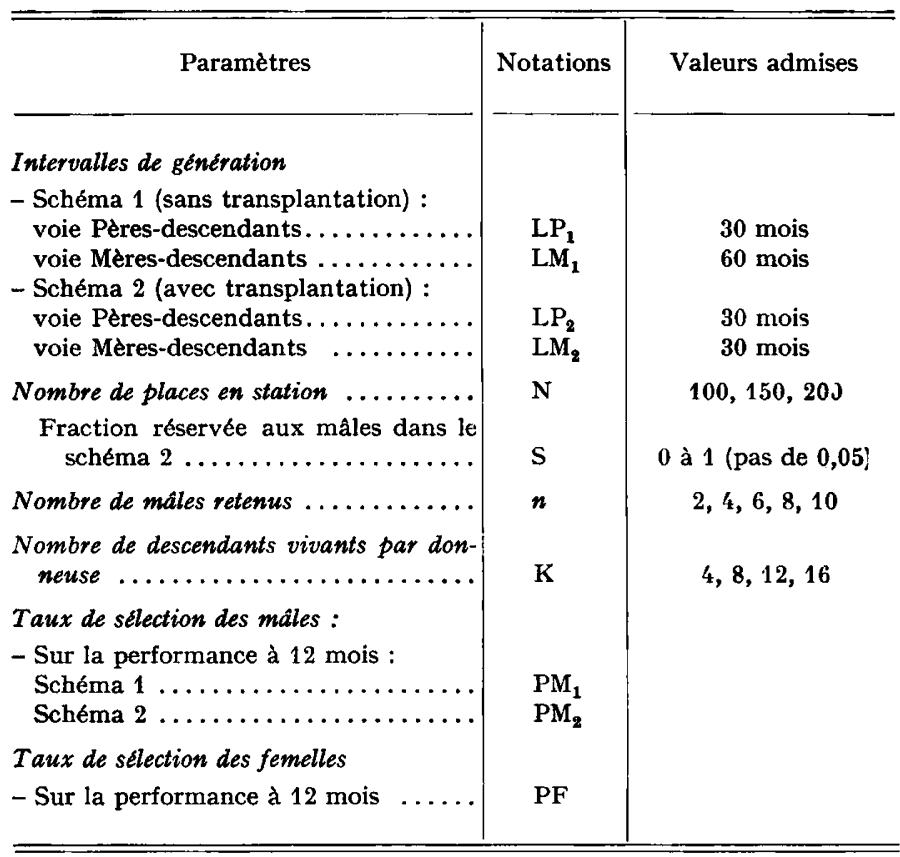

\section{2. - Méthodes et critères de comparaison}

Comme l'ont fait LAND et Hirl (1975), nous comparerons les deux schémas envisagés et leurs variantes sur la base du progrès génétique qu'ils permettent d'espérer et de l'accroissement annuel du taux de consanguinité qu'ils supposent.

On désignera par $\mathrm{PM}_{1}=n / \mathrm{N}$ et $\mathrm{PM}_{2}=n / \mathrm{NS}$ les taux de sélection des mâles pour chacun des schémas et par $\mathrm{PF}=n^{\prime} / \mathrm{N}(\mathrm{r}-\mathrm{S})$ le taux de sélection des femelles dans le second cas.

Les expressions simplifiées du progrès génétique s'écrivent alors :

$$
\Delta g_{1} \propto \frac{i\left(\mathrm{PM}_{1}\right)}{\mathrm{LP}_{1}+\mathrm{LM}_{1}} \quad \text { et } \quad \Delta g_{2} \propto \frac{i\left(\mathrm{PM}_{2}\right)+i(\mathrm{PF})}{\mathrm{LP}_{2}+\mathrm{LM}_{2}}
$$


où $i\left(\mathrm{PM}_{1}\right)$ est l'intensité de sélection correspondant au taux $\mathrm{PM}_{1}$. Ces valeurs sont en effet suffisantes pour comparer les deux schémas dans la mesure où héritabilité et écart-type du critère de sélection sont supposés identiques pour les deux sexes. Les intensités de sélection utilisées sont calculées par la formule de Burrows (1972) pour tenir compte des effectifs limités en cause dans chaque situation. On notera à ce propos que le nombre $n^{\prime}$ de femelles retenues doit être suffisant pour assurer le renouvellement; on aura donc $\frac{n^{\prime} K}{2}=N(x-S)$ si $S$ est inférieur à $r / 2$ et $n^{\prime} \mathrm{K} / 2=\mathrm{NS}$ dans le cas contraire. Il en résulte que le progrès génétique considéré comme une fonction de $\mathrm{S}$, dans le cas du second schéma, aura deux expressions différentes $\left(f_{1}\right.$ et $\left.f_{\mathrm{g}}\right)$ selon que la fraction $S$ des places de station réservées au contrôle de mâles sera ou non supérieure à $\mathrm{I} / 2$.

Pour ce qui concerne l'accroissement annuel du taux de consanguinité, second critère de comparaison des schémas étudiés, son expression sera dérivée de celle de l'effectif génétique donnée par LATTER (1959). Dans le cas particulier où le nombre de descendants par reproducteur est une variable aléatoire, cette expression est la suivante :

$$
\Delta \mathrm{F}=\frac{\mathrm{I}}{4 \overline{\mathrm{L}}^{2}}\left(\frac{\mathrm{I}}{\mathrm{N} \delta}+\frac{\mathrm{I}}{\mathrm{N} \%}\right)
$$

où $\overline{\mathrm{L}}$ représente l'intervalle de génération moyen et $\mathrm{N} \delta$ et $\mathrm{N}$ 早 respectivement les nombres de reproducteurs de chacun des sexes sélectionnés par an. Dès lors les expressions particulières pour chacun des schémas sont :

et

$$
\begin{aligned}
& \Delta \mathrm{F}_{1} \simeq \mathrm{I} / n\left(\mathrm{LP}_{1}+\mathrm{LM}_{1}\right)^{2} \\
& \Delta \mathrm{F}_{2}=\left(\mathrm{I} / n+\mathrm{I} / n^{\prime}\right) /\left(\mathrm{LP}_{2}+\mathrm{LM}_{2}\right)^{2}
\end{aligned}
$$

\section{II. - RÉSULTATS}

Dans le but de mieux cerner l'efficacité relative de chacun des schémas étudiés un certain nombre de paramètres ont été considérés comme variabiles soit parce que leur valeur pouvait résulter de contraintes techniques (tel $\mathrm{K}$ par exemple) ou financières (tel $\mathrm{N}$ ) soit parce qu'ils pouvaient être modifiés à loisir (tels $n$ et $\mathrm{S}$ ). Il résulte de cette situation que les deux schémas ne-peuvent être comparés simplement sans une analyse préalable de l'incidence de ces paramètres sur la valeur de chacun des deux critères d'efficacité et sans la recherche des combinaisons optimales pour chacun d'eux.

\section{I. - Recherche des situations optimales}

Le tableau 2 indique la valeur des variables optimisant chacun des deux critères d'efficacité des schémas. Il indique en particulier que seul l'accroissement du nombre de places en station permet à la fois d'accroître le progrès génétique et de réduire l'augmentation du taux de consanguinité. Les variations de tous les autres paramètres ont des effets antagonistes sur les deux critères d'efficacité génétique des schémas. Dès lors seule la connaissance de pondérations économiques pour chacun des deux critères permettrait de définir les conditions optimales de réalisation de chacun des schémas.

Dans l'ignorance de ces pondérations on doit se borner à indiquer, pour chaque valeur fixée de l'un des critères, la combinaison des variables conduisant à la valeur optimale de l'autre critère.

Dans le cas du premier schéma étudié et pour une capacité de testage $(\mathrm{N})$ donnée les valeurs respectives du progrès génétique et de l'accroissement de consanguinité $\left(\Delta g_{1}\right.$ et $\left.\Delta F_{1}\right)$ ne dépendent que du nombre $n$ de mâles sélectionnés.

Dans le cas du schéma avec transplantation, même si les deux paramètres de contrainte ( $\mathrm{N}$ et $\mathrm{K}$ ) sont fixés, il convient de rechercher les combinaisons optimales 
de $n$ et $\mathrm{S}$ pour toute valeur donnée de l'un des critères d'efficacité. Cette recherche peut notamment consister à étudier les variations de chacun des critères avec une des variables, $\mathrm{S}$ par exemple, pour une valeur donnée de l'autre variable, $n$ dans ce cas.

\section{TABLEAU 2}

Valeurs des paramètres optimisant les critères de comparaison des schémas

Values of the parameters optimizing the criteria used to compare the situations

\begin{tabular}{|c|c|c|c|c|}
\hline \multirow{3}{*}{ Paramètre } & \multicolumn{4}{|c|}{ Critère } \\
\hline & \multicolumn{2}{|c|}{$\begin{array}{c}\text { Schéma 1 } \\
\text { (sans transplantation) }\end{array}$} & \multicolumn{2}{|c|}{$\begin{array}{c}\text { Schéma 2 } \\
\text { (avec transplantation) }\end{array}$} \\
\hline & $\begin{array}{l}\text { Progrès génétique } \\
\qquad \Delta g_{1}\end{array}$ & $\begin{array}{c}\text { Accroissement de } \\
\text { consanguinité } \\
\Delta \mathrm{F}_{1}\end{array}$ & $\begin{array}{l}\text { Progrès génétique } \\
\qquad \Delta g_{2}\end{array}$ & $\begin{array}{c}\text { Accroissement de } \\
\text { consanguinité } \\
\qquad \Delta \mathbf{F}_{\mathbf{2}}\end{array}$ \\
\hline Capacité de testage $(\mathrm{N})$ & Maximum & Indépendant & Maximum & Maximum \\
\hline $\begin{array}{l}\text { Nombre de } \\
\text { mâles retenus }(n)\end{array}$ & Minimum & Maximum & Minimum & Maximum \\
\hline $\begin{array}{c}\text { Nombre de descendants } \\
\text { par femelle (K) }\end{array}$ & & & Maximum & Minimum \\
\hline $\begin{array}{c}\text { Fraction de } \mathrm{N} \text { réservée } \\
\text { aux mâles (S) }\end{array}$ & & & $1 / 2$ à 1 & 0 ou 1 \\
\hline
\end{tabular}

On notera à ce propos que aussi bien $\mathrm{S}$ que $n$ ne peuvent prendre que des valeurs discrètes. Dès lors la variation de chacun des critères $\Delta g_{2}$ et $\Delta \mathrm{F}_{3}$, sera elle-même discontinue. Ainsi $n$ ne peut prendre que les valeurs entières comprises entre I et $\frac{\mathrm{NK}^{\prime}}{2+\mathrm{K}}$ et $\mathrm{S}$ peut prendre les valeurs comprises entre $\frac{n}{\mathrm{~N}}$ et $\frac{\mathrm{K}}{2+\mathrm{K}}$ qui correspondent aux valeurs entières du nombre de mâles en station et du nombre de places. En particulier $S$ est supérieur à $I / 2$ dès que $n$ est plus grand que $\frac{N}{2}$ puisque dans ce cas la moitié au moins des places de station est nécessaire au contrôle des mâles sélectionnés. $\mathrm{L} a$ valeur minimale de $\mathrm{S}$ correspond à la non sélection des mâles contrôlés et sa valeur maximale à la non sélection des femelles contrôlées.

On simplifiera l'exposé en considérant les variations des critères comme continues pour une valeur fixée de $n$. Ceci revient à admettre une variation continue de $\mathrm{S}$, hypothèse réaliste dès que la capacité de testage $\mathrm{N}$ est assez grande.

Variations de $\Delta g_{2}$ en fonction de $S$ à $\mathbf{n}$ fixé.

Nous avons déjà mentionné l'existence de deux expressions, $f_{1}$ et $f_{2}$, du progrès génétique selon que $S$ est ou non inférieur à $I / 2$. La première est toujours croissante, alors que la seconde passe par un maximum pour une valeur de $S$ qui augmente avec le nombre de mâles $(n)$ retenus. 
Il existe ainsi un nombre $\hat{n}$ de mâles sélectionnés en dessous duquel le maximum $f_{2}$ est atteint pour des valeurs de $S$ inférieures à $\mathrm{I} / 2$, donc hors de 1'intervalle de définition de cette fonction. Dans ce cas le progrès génétique est toujours maximum pour $\mathrm{S}=\mathrm{I} / 2$, par contre pour les valeurs de $n$ supérieures à $\hat{n}$ le maximum de $\Delta g_{2}$ correspond au maximum de $f_{2}$ qui est atteint pour des valeurs de $S$ supérieures à $I / 2$.

Variations de $\Delta \mathrm{F}_{2}$ en fonction de $S$ à $\mathrm{n}$ fixé.

Dans le cas où $n$ est fixé $\Delta \mathrm{F}_{\mathrm{z}}$ varie avec $\frac{\mathrm{I}}{n^{\prime}}$ donc avec $\frac{\mathrm{I}}{\mathrm{I}-\mathrm{S}}$ ou $\frac{\mathrm{I}}{\mathrm{S}}$ selon que $\mathrm{S}$ est ou non supérieur à $I / 2$ de telle sorte que l'accroissement du taux de consanguinité est d'autant plus grand que $S$ tend vers $x / 2$, et maximum pour cette valeur dans tous les cas où elle est possible c'est-à-dire quand $n$ est compris entre I et $\frac{\mathrm{N}}{2}$, pour la valeur minimale de $\mathrm{S}$ dans les autres cas.

En résumé et comme nous l'avons déjà mentionné les valeurs maximales du progrès génétique (obtenues la plupart du temps pour $S=\frac{I}{2}$ et $P F=\frac{2}{K}$ ) correspondent en général aux situations les plus défavorables pour l'accroissement du taux de consanguinité $\left(\Delta \mathrm{F}_{2}\right.$ maximum). Dès lors seule l'analyse des variations conjointes de $\Delta g_{2}$ et $\Delta \mathrm{F}_{2}$ avec $\mathrm{S}$ et $n$ peut permettre d'appréhender les combinaisons optimales de ces deux paramètres à savoir celles qui maximisent le progrès génétique pour chaque valeur de l'augmentation annuelle du taux de consanguinité.

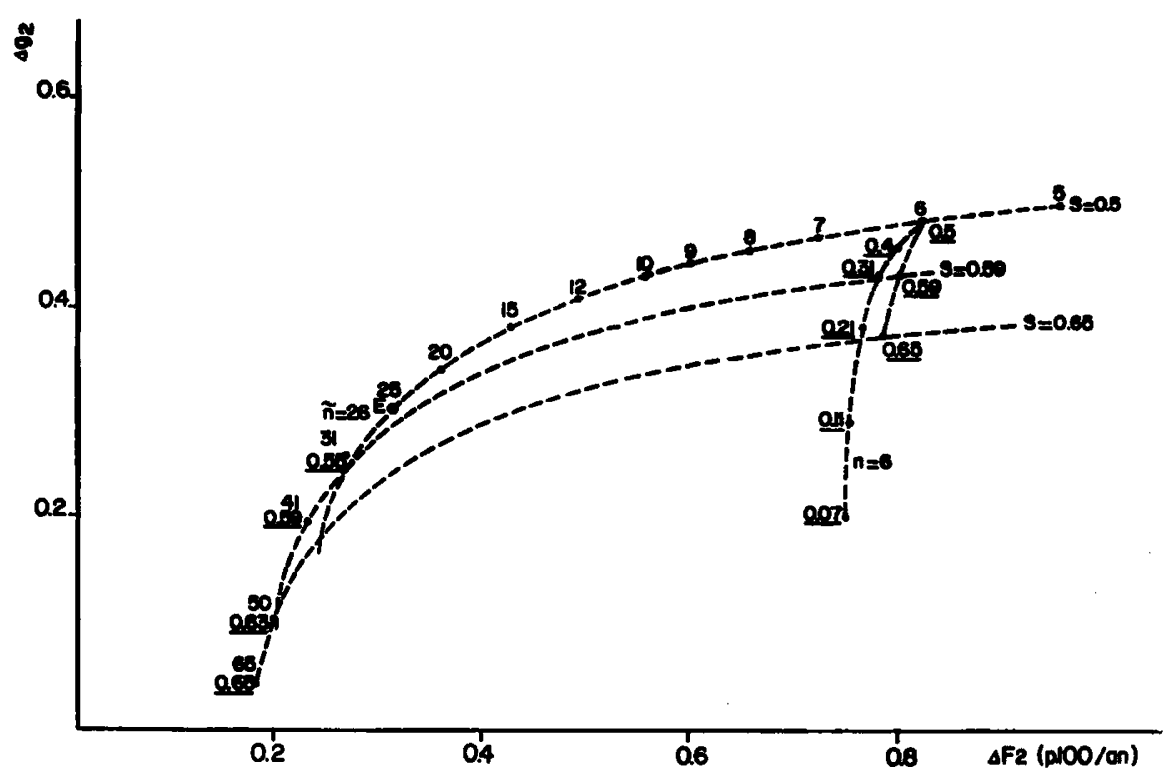

Fig. 2. - Valeurs du progrès génétique $\Delta \mathrm{g}_{2}$ et de l'accroissement du taux de consanguinité $\Delta \mathrm{F}_{2}$ en fonction de la part $\mathrm{S}$, des $\mathrm{N}$ places en station, rêservée aux mâles et du nombre $\mathrm{n}$ de mâles retemus

(roo places en station, 4 descendants par donneuse)

Values of genetic progress $\Delta g_{2}$ and of increase of inbreeding rate $\Delta \mathrm{F}_{2}$ in terms of the part $\mathrm{S}$ of $\mathrm{N}$ places in the station reserved for males and of the $\mathrm{n}$ number of males kept (roo places in the station, 4 progeny per donor) 
Variations conjointes de $\Delta g_{2}$ et $\Delta \mathrm{F}_{2}$ avec $S$ et $\mathrm{n}$.

Nous esquisserons cette analyse à partir de la représentation graphique du cas particulier où $\mathrm{N}=$ Ioo et $\mathrm{K}=4$ (fig. 2).

Pour chaque valeur fixée de $n$ entière, on obtient un ensemble de points correspondant aux valeurs de $\Delta g_{2}$ en fonction de $\Delta \mathrm{F}_{2}$ pour les différentes valeurs possibles de $\mathrm{S}$.

La courbe joignant les points correspondants à ces valeurs de $S$ pour $n=6$ est représentée sur la figure et illustre les variations de $\Delta g_{2}$ et $\Delta \mathrm{F}_{2}$ avec $\mathrm{S}$ à $n$ fixé, mentionnées plus haut. L'ensemble des courbes de ce type pour chaque valeur de $n$ représente l'ensemble des situations possibles. Parmi ces nombreuses situations seules sont intéressantes les. combinaisons de $\mathrm{S}$ et $n$ qui autorisent un progrès génétique maximum pour une valeur fixée de l'accroissement du taux de consanguinité.

On peut calculer en admettant $n$ et $\mathrm{S}$ divisibles une enveloppe de toutes ces courbes qui représenterait dans ce cas l'ensemble des situations optimales pour $n$ et pour S. Une telle enveloppe peut également être appréhendée graphiquement en joignant les points correspondant à une valeur fixée de $S$ pour les différentes vaieurs de $n$. Les courbes $\mathrm{S}=0,65 ; 0,59$; et 0,50 sont représentées sur la figure 2 . Leur examen appelle trois remarques :

- L'enveloppe, lieu des solutions optimales ne concerne que les valeurs de $\mathrm{S}$ supérieures ou égales à 0,5 .

- Il existe sur cette courbe un point $\mathrm{E}$ tel que à partir de ce point les solutions de la partie droite de la courbe correspondent à $n$ décroissant $<\tilde{n}$ (valeur optimale en ce point $\mathrm{E}$ ) et $\mathrm{S}=0,5$ alors que les solutions de la partie gauche correspondent à $n$ croissant $>\tilde{n}$ et à $\mathrm{S}$ croissant de 0,5 à $\frac{\mathrm{K}}{2+\mathrm{K}}$. Dans le cas particulier du graphique $\tilde{n}=26$ et la valeur optimale de $S$ varie de 0,50 à 0,65 .

- Les solutions réelles et optimales correspondent en fait à l'ensemble des points situés soit sur l'enveloppe précédemment définie, soit immédiatement en dessous étant donné que les valeurs discontinues prises à la fois par $n$ et par $\mathrm{S}$ entrâ̂nent des valeurs discontinues aussi bien pour $\Delta g_{2}$ que pour $\Delta \mathrm{F}_{2}$. En particulier il existe un certain nombre de solutions acceptables pour lesquelles $S$ est légèrement inférieur à $\mathrm{I} / 2$ par exemple les solutions $n=6$ et $\mathrm{S}=0,44 ; 0,46 ; 0,48$, qui conduisent à un progrès génétique supérieur à la solution $n=7$ et $\mathrm{S}=0,5$ mais pour des valeurs plus grandes de l'accroissement de consanguinité.

Cependant il nous a semblé possible pour la suite de l'analyse de comparer les différentes variantes du schéma avec transplantation ( $\mathrm{N}$ et $\mathrm{K}$ variables) sur la base de cette courbe enveloppe qui représente en fait une légère surestimation des solutions optimales réalisables. Dans chaque cas il semble intéressant de préciser le nombre $\tilde{n}$ de mâles retenus à partir duquel la fraction optimale du nombre de places de station réservée aux mâles n'est plus $\mathrm{I} / 2$, et l'évolution de cette fraction avec $n$ qui nous semble le paramètre de choix le plus important tant vis-à-vis de $\Delta g_{2}$ que de $\Delta \mathrm{F}_{2}$.

\section{2. - Efficacité comparée des schémas}

Nous avons représenté graphiquement sur la figure 3 et pour $\mathrm{N}=$ roo places de station, les courbes d'évolution conjointe du progrès génétique et de l'accroissement du taux de consanguinité en fonction de $n$ dans le cas du premier schéma, en 
fonction des combinaisons optimales de $n$ et $\mathrm{S}$ (supposés continus) dans le cas du schéma avec transplantation pour plusieurs valeurs de $K(4,8$, I2 et 16 descendants par femelle donneuse). Le nombre $\tilde{n}$ de mâles correspondant au point E est mentionné sur les courbes.

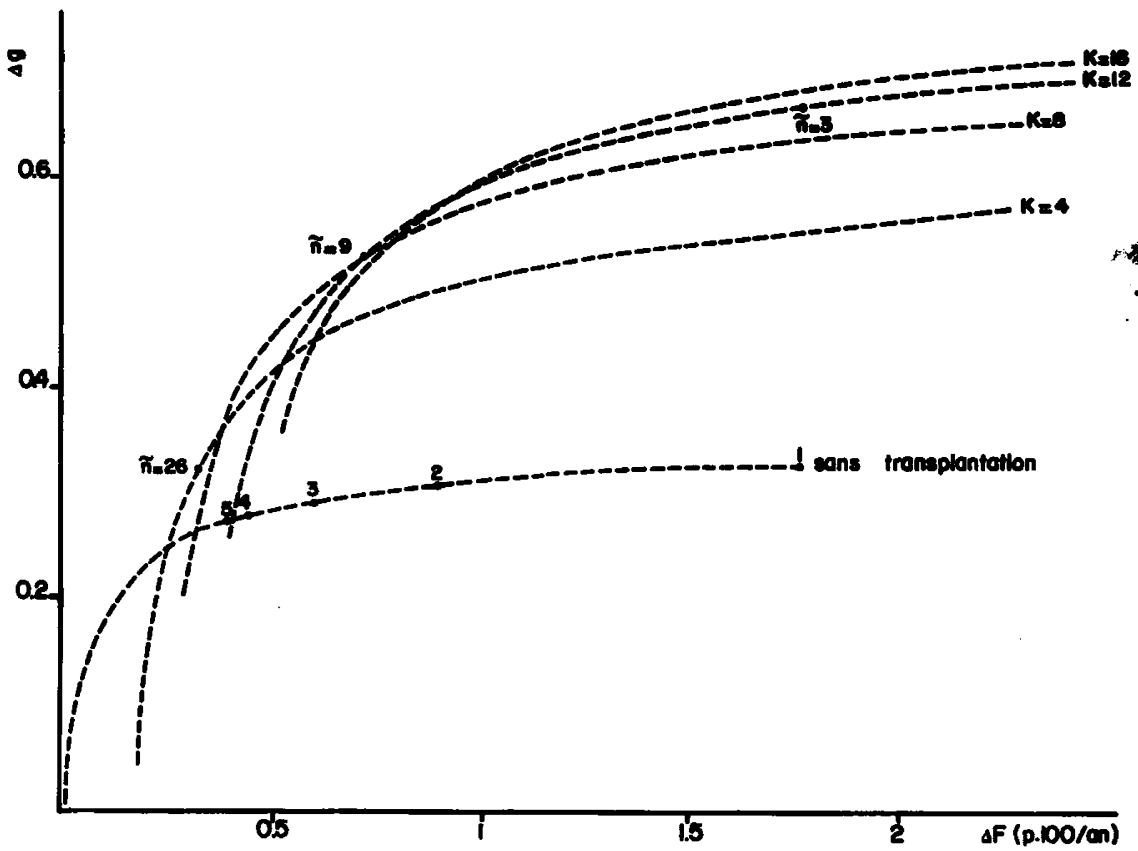

FIG. 3. - Valeurs des progrès génétiques $\Delta g_{1}$ et $\Delta g_{2}$ et des accroissements du taux de consanguinité $\Delta \mathrm{F}_{1}$ et $\Delta \mathrm{F}_{2}$, pour chacun des 2 schémas, en fonction du nombre de males retenus $\mathrm{n}$, et pour le second schema de la part $\mathrm{S}$ des $\mathrm{N}$ places en station réservée aut máles et du nombre de descendants $\mathrm{K}$ par donneuse $(\mathrm{N}=\mathrm{IOO})$.

Values of genetic progress, $\Delta g_{1}$ and $\Delta g_{2}$, and of inbreeding rate increases, $\Delta \mathrm{F}_{1}$ and $\Delta \mathrm{F}_{2}$, for each of the two situations, according to the number $\mathrm{n}$ of males kept, and, for the second situation, according to the part $\mathrm{S}$ of the $\mathrm{N}$ places in the station reserved for males and to the number $\mathbf{k}$ of progeny per donor cow $(\mathrm{N}=100)$.

Ces différentes courbes appellent un certain nombre de commentaires.

En premier lieu elles indiquent que l'évolution du progrès génétique avec l'accroissement de consanguinité obéit à la loi des rendements décroissants, de telle sorte que l'acceptation d'une augmentation annuelle de I p. Ioo du taux de consanguinité permet d'espérer 75 à $95 \mathrm{p}$. Ioo du progrès génétique maximum selon les cas et qu'au-delà de 2 p. Ioo par an le progrès génétique n'augmente pratiquement plus. En l'absence de pondérations économiques pour ces deux critères antagonistes il semble prudent de limiter cet accroissement de la consanguinité à des valeur inférieures à I p. Ioo par an.

Dans ces conditions on constate en second lieu que pour des valeurs autorisées faibles de $\Delta \mathrm{F}$ (inférieures à $0,3 \mathrm{p}$. Ioo par an dans notre cas, qui correspondent à plus de 6 mâles retenus pour le schéma classique et plus de 20 pour le schéma avec transplantation) la pratique de la transplantation d'œufs ne présente pas d'intérêt pour la sélection considérée. Par contre, elle permet environ le double de progrès génétique dès qu'on admet un accroissement annuel de consanguinité de l'ordre de $I, 5$ p. Ioo. 
Ensuite il semble, au moins dans le cadre de la sélection envisagée, que l'intérêt de la transplantation ne réside pas dans l'obtention d'un nombre élevé d'œufs transplantés par femelle donneuse puisque au-delà de 6 à 8 descendants par donneuse le progrès génétique n'augmente pratiquement plus, même pour des accroissements importants de la consanguinité et que par contre les accroissements faibles ne sont plus possibles. I1 faut à ce propos remarquer que nous obtenons là encore un faisceau de courbes, en fonction de $K$, admettant une enveloppe telle que pour chaque valeur fixée de l'un des deux critères, il existe une solution optimisant l'autre, solution qui est une combinaison optimale des trois paramètres $n, \mathrm{~S}$ et $\mathrm{K}$.

Nous noterons enfin que dans le cas du second schéma la part de la capacité de testage à réserver aux mâles est d'autant plus grande qu'on retient plus de mâles et que le nombre de descendants par femelle donneuse augmente.

\section{III. - DISCUSSION ET CONCLUSION}

Il est bien évident que notre étude, comme toute étude théorique de prévison, n'échappe pas à la constatation que les résultats obtenus dépendent dans une large mesure des hypothèses de base du modèle et de la valeur des paramètres utilisés, que ces résultats ne sont, par ailleurs, pas directement transposable à d'autres situations.

Ainsi, dans le cas du schéma avec transplantation la sélection s'opère sur des groupes de pleins frères - pleines sœurs et il serait intéressant d'analyser les conséquences d'autres types de sélection que la sélection massale (familiale, combinée, etc.) tant sur l'espérance du progrès génétique que sur la réduction d'effectif génétique, en particulier si le nombre de parents sélectionnés est faible. Einfin, et dans la mesure où la fécondation in vitro des œufs n'est pas envisagée, il conviendrait de ne pas considérer les nombres de mâles et de femelles retenus comme indépendants. Les solutions conduisant à un nombre de mâles sélectionnés supérieur au produit du nombre de femelles donneuses par le nombre de cycles de transplantation par femelle ne sont pas admissibles par exemple.

Nous sommes également conscients du fait que les deux critères d'efficacité utilisés sont insuffisants et qu'il conviendrait de comparer les différents schémas en tenant compte des coûts d'entretien des femelles reproductrices selon leur effectif et le fait qu'il s'agisse de femelles de race pure à viande ou de femelles réceptrices. Les coûts de superovulation et de transplantation ne sont probablement pas négligeables non plus. Nous avons, en l'absence de ces valeurs, pratiquement admis que la réduction des premiers était compensée par les seconds, c'est-à-dire que les coûts de production des $\mathrm{N}$ veaux entrés en station étaient équivalents dans les différentes situations ce qui de toute évidence ne peut être vérifié.

En tout état de cause, cette étude partielle illustre assez bien l'intérêt et les limites des techniques nouvelles de maîtrise de la reproduction vis-à-vis de l'amélioration génétique des caractères classiques de production de viande. On peut en retenir que l'utilisation de la transplantation d'œufs pour la sélection sur la croissance ne se justifie que si on accepte corollairement un accroissement du taux de consanguinité de plus de $0,5 \mathrm{p}$. Ioo par an. Dans ce cas le progrès génétique peut être doublé et on 
doit accorder d'autant plus d'importance à la sélection des femelles qu'elles produisent davantage de descendants et qu'on admet une augthentation importante du taux de consanguinité. On notera également que dans la plupart des cas il convient d'utiliser les capacités de testage pour contrôler mâles et femelles avec la même importance. On retrouve alors les conditions et le schéma de sélection envisagés par LAND et HILI, (I975) et il faut remarquer que cette situation correspond également à l'entretien d'un nombre minimum de femelles donneuses et surtout de femelles réceptrices. Enfin l'intérêt génétique de la transplantation est surtout sensible pour un faible nombre d'œufs transplantés par donneuse, ce qui dans l'état actuel de ces techniques est le plus vraisemblable. Ceci ne laisse rien présumer par contre sur l'intérêt de la transplantation ou même d'un nombre plus élevé d'œufs dans d'autres situations, par exemple pour la sélection d'autres caractères ou pour le contrôle de descendance des femelles ou bien encore tout simplement dans le but d'accroître la production de viande ; toutes situations nouvelles qu'il conviendra d'envisager à terme et dans la mesure où se confirmera la possibilité d'utiliser largement ces nouvelles techniques.

Ręu pour publication en mai 1976.

\title{
REMERCIEMENTS
}

Nous remercions R. B. LAND pour la lecture critique de notre manuscrit.

\section{SUMMARY}

\section{EGG TRANSFER AS A METHOD FOR INCREASING SELECTION EFFICIENCY}

\author{
IN BEEF CATTLE
}

New techniques of superovulation and egg transfer are studied as a method for increasing genetic progress in a beef cattle line to be used in terminal crossing, when breeding animals are chosen on individual performance testing in a station where the number of animals tested is limited. Two situations are compared :

- a reference situation without transfer where only the males are tested,

- a situation with transfer where some places are reserved for female testing.

Variable numbers of males and females tested and selected as breeding animals are compared for different testing facilities. For the second situation, we also looked at the oportunity to obtain a more or less large mean number of progeny per donor female.

The different situations thus simulated at a fixed testing capacity are compared using the maximum genetic progress which may be expected for a given increase of the inbreeding rate. Our results lead to the following conclusions :

- transfer is particularly advantageous for mid-values of inbreeding rate increase since between 0.5 and I.5 p. Ioo per year genetic progress may be doubled as compared to the reference situation. On the other hand, the situation without transfer is preferable below $0.5 \mathrm{p}$. 100 ; when the annual increase in inbreeding rate is more than $1.5 \mathrm{p}$. Ioo there is no significant augmentation in relative genetic progress.

- genetic advantage of transfer is only really significant for limited numbers of transferred eggs since relative increase in genetic progress is very low when more then 6 to 8 progeny are obtained per donor female ;

- the optimal number of places to reserve in the station for male testing is always equal to or higher than $50 \mathrm{p}$. Ioo and increases with the number of selected bulls. 


\section{RÉFÉRENCES BIBLIOGRAPHIQUES}

Burrows P. M., 1972. Expected selection differentials for directional selection, Biometrics, 28, ro91Iroo.

Cunningham E. P., r974. New developments in reproductive physiology and their consequences for animal breeding. Symposium en l'honneur du $25^{\mathrm{e}}$ anniversaire du centre d'insémination de Neustadt a.d. Aisch, avril r974, p. 30.

LAND R.B., HiLl W. G., I975. The possible use of superovulation and embryo transfer in cattle to increase response to selection. Anim. Prod., 21, I-I2.

LATter B. DH., 1959. Genetic sampling in a random mating control population of constant size and sex ratio. Aust. J. Biol. Sci., 12, 500-505.

Mocquot J.-C., Foulley J.-L., r973. Recherche des conditions de rentabilité d'un schéma de sélection d'une souche de bovins destinés au croisement de première génération pour la production de veaux de boucherie. Ann. Génet. Sél. anim., 5, 189-209.

Vissac B., r974. Possibilité de valorisation des techniques de transplantation d'cufs au niveau de l'amélioration des productions bovines. Polycopié, pp. 7. 